\title{
Shared neuronal bases of inhibition and economic choice in orbitofrontal cortex
}

\author{
Pragathi Priyadharsini Balasubramani ${ }^{1} \&$ Benjamin Y. Hayden ${ }^{2}$
}

1. Department of Psychiatry, University of California San Diego, La Jolla, CA. 92037

2. Neuroscience and Center for Magnetic Resonance Research, University of Minnesota

Minneapolis, MN. 55455

Abbreviated title: Stopping and choice in OFC ensembles

Corresponding Author:

Pragathi Priyadharsini Balasubramani

Department of Psychiatry, University of California San Diego, La Jolla, CA. 92037

Email: pragathipriyadharsini@gmail.com

Acknowledgements: This work was supported by an R01 (DA038615) to BYH. We thank Meghan C. Pesce for help with data collection.

The authors declare no conflict of interest. 


\section{ABSTRACT}

35 Economic choice and inhibition are two important elements of our cognitive repertoires

36 that may be closely related. We and others have noted that during economic choice, options are

37 typically considered serially; this fact provides important constraints on our understanding of

38 choice. Notably, asynchronous contemplation means that each individual option is subject to an

39 accept-reject decision. We have proposed that these component accept-reject decisions may have

40 some kinship with stopping decisions. One prediction of this idea is that stopping and choice may

41 reflect similar neural processes occurring in overlapping brain circuits. To test the idea, we

42 recorded neuronal activity in orbitofrontal cortex (OFC) Area 13 while macaques performed a

43 stop signal task interleaved with a structurally matched choice task. Using neural network

44 decoders, we find that OFC ensembles have overlapping codes for stopping and choice: the

45 decoder that was only trained to identify accept vs. reject trials performed with higher efficiency

46 even when tested on the stop trials. These results provide tentative support for the idea that

47 mechanisms underlying inhibitory control and choice selection may be subject to theoretical

48 unification. 


\section{INTRODUCTION}

51 Foraging theory is a branch of behavioral ecology centered on understanding the

52 psychology of reward-based choices in natural contexts (Stephens and Krebs 1986). It

53 provides a unique perspective on many aspects of economic choice and has recently

54 come to influence the neuroscience and psychology of decision-making (Pearson et al.

55 2014; Calhoun and Hayden 2015; Mobbs et al. 2018; Hayden et al., 2011). A core idea in

56 foraging theory is that natural decisions are fundamentally structured around accepting

57 vs. rejecting - taking or passing up a single option that is the sole or primary focus of

58 attention (Shapiro et al. 2008; Vasconcelos et al. 2010; Kacelnik et al. 2011; Pirrone et al.

59 2017; Ojeda et al. 2018). Even ostensibly binary economic choices, in this view, reflect a

60 pair of (potentially interacting) accept-reject choices. Accepting involves selecting the

61 attended or activated option, or, more abstractly, performing the afforded action (Cisek

62 and Kalaska 2010; Cisek and Pastor-Bernier 2014; Hayden and Moreno-Bote 2018).

63 Rejecting involves countermanding the afforded action. An ostensibly binary economic

64 choice, then, may be seen as two related decisions about whether to go or stop choosing

65 the attended option or the afforded action (Krajbich et al. 2010; Kacelnik et al. 2011;

66 Hayden 2018).

67 Here we consider one particular implication of this foraging-inspired line of work:

68 that economic choice may be subject to theoretical unification with stopping decisions

69 (Hayden 2018; Balasubramani et al. 2019). The rationale for this idea is straightforward.

70 Both economic and stopping decisions involve a single option as an offer and a choice of

71 whether to pursue that option or refrain from pursuing it. Both are framed around a 
72 default option (accepting and going, respectively) and a non-default (rejecting and

73 stopping), that is, deliberate inhibition of the default option.

74 There is at least some evidence in support of this hypothesis. First, stopping and

75 economic choice tend to activate a similar set of brain regions, including the pre-motor

76 cortex, the ventrolateral prefrontal cortex, basal ganglia, and the thalamus (Schall et al.

77 2002; Aron and Poldrack 2006; Aron 2007; Sakagami and Pan 2007; Cisek and Kalaska

78 2010; Cisek 2012; Hampshire and Sharp 2015). Second, this way of looking at choice is

79 consistent with some recent studies that suggest that binary choice involves a serial, not

80 parallel, consideration of options (Krajbich et al. 2010; Strait et al. 2014; Rich and Wallis

81 2016; reviewed in Hayden and Moreno-Bote, 2018). These studies and others indicate

82 that attention is largely limited to a single option, which is evaluated, often relative to the

83 other one (Lim et al. 2011; Rudebeck and Murray 2014; Strait et al. 2014; Strait et al.

84 2015; Rich et al. 2017; Xie et al. 2018).

85 We recorded neuronal activity in orbitofrontal cortex (OFC) area 13. The

86 importance of OFC for economic choice is largely undisputed, although its specific role

87 remains to be determined (Wallis 2007; Schoenbaum et al. 2009; Padoa-Schioppa 2011;

88 Rudebeck and Murray 2014; Wilson et al. 2014; Rich et al. 2017). It is clear, nonetheless,

89 that activity of OFC correlates with the values of offers and of chosen options, and is

90 likely to be critical for value comparison as well (Padoa-Schioppa and Assad 2006;

91 Padoa-Schioppa 2013; Raghuraman and Padoa-Schioppa 2014; Wallis, 2007). In contrast

92 to its clear role in choice, the contribution of the OFC to stopping remains to be

93 determined. Specifically, some studies and surveys argue against its inhibitory role

94 (Schoenbaum et al. 2003; Chudasama et al. 2006; Ghods-Sharifi et al. 2008; Rudebeck 
95 and Murray 2014; Stalnaker et al. 2015). Others argue in support of some inhibitory role

96 (Mishkin 1964; Iversen and Mishkin 1970; Dias et al. 1996; Roberts and Wallis 2000;

97 Horn et al. 2003; Eagle et al. 2007; Chikazoe et al. 2009; Majid et al. 2013; Bryden and

98 Roesch 2015; Balasubramani et al. 2019). Our previous study on the topic suggests that it

99 does contribute to inhibition/stopping, although this is part of its more complex role in

100 setting the stage for action, which includes its economic functions (Balasubramani et al., 101 2019).

102 Here we sought to test one specific prediction of the overlap hypothesis by

103 comparing neuronal activity in a choice task with that observed in a stopping task. For

104 stopping task, we use the stop signal task framework, a standard tool for studies of

105 stopping and inhibitory control (Logan and Cowan 1984; Logan 1994; Hanes and Schall

106 1995). We then designed a novel economic choice task that follows, as closely as

107 possible the design of the stopping task and call it choice signal task, so that we could

108 focus on differences relevant to our hypothesis. 


\section{METHODS}

112 Some of the data presented here were analyzed for a previous manuscript

113 (Balasubramani et al., 2019). Specifically, data from the stopping task were analyzed in

114 that manuscript. Data from the choice task were collected in the same sessions but not

115 analyzed therein.

\section{Subjects}

117 Two male rhesus macaques (Macaca mulatta, subject $\mathrm{J}$, age 10, and subject T, age 118 5) served as subjects. All animal procedures were approved by the University Committee 119 on Animal Resources at the University of Rochester and were designed and conducted in 120 compliance with the Public Health Service's Guide for the Care and Use of Animals.

\section{Recording site}

A Cilux recording chamber (Crist Instruments) was placed over the Area 13 of OFC, as defined by (Paxinos and Watson 2006). The targeted area expands along the coronal planes situated between 28.65 and $33.60 \mathrm{~mm}$ rostral to the interaural plane with varying depth. Position was verified by magnetic resonance imaging with the aid of a Brainsight system (Rogue Research Inc). Neuroimaging was performed at the Rochester Center for Brain Imaging, on a Siemens 3T MAGNETOM Trio Tim using $0.5 \mathrm{~mm}$ voxels. We confirmed recording locations by listening for characteristic sounds of white and grey matter during recording, which in all cases matched the loci indicated by the Brainsight system.

\section{Electrophysiological techniques}

Single electrodes (Frederick Haer \& Co., impedance range 0.8-4 MOhm) were lowered using a microdrive (NAN Instruments) until waveforms of between one and five neuron(s) were isolated. Individual action potentials were isolated on a Plexon system. Neurons were selected for study solely based on the quality of isolation; we never preselected neurons based on task-related response properties. The number of neurons to be collected was determined a priori based on exploratory analyses of previously collected datasets and was not adjusted during recording based on analyses performed mid-experiment. 146 and confirmed before, during, and after recording.

\section{Task}

Eye position was sampled at $1,000 \mathrm{~Hz}$ by an infrared eye-monitoring camera system (SR Research). Stimuli were controlled by a computer running MATLAB (Mathworks) with Psychtoolbox (Brainard and Vision 1997) and Eyelink Toolbox (Cornelissen et al. 2002). A standard solenoid valve controlled the duration of water delivery. The relationship between solenoid open time and water volume was established 
The stop signal task (Figure 1A) is a measure of self-control that provides an alternative approach that avoids some of the limitations of intertemporal choice tasks (Hayden 2016). The task followed standard stop signal paradigm (Logan and Cowan 1984; Logan 1994; Hanes and Schall 1995). Subjects were placed in front of a computer monitor (1920x1080 pixels) with black background. Following a brief (300 msec) central fixation on a white circle (radius 25 pixels, Figure 1A), the fixation spot disappeared on the appearance of eccentric saccade target (90px white square, 2.38 degrees, positioned at 288 pixels, 7.62 degrees, in left or 1632 pixels, 43.18 degrees, in right of screen, $50 \%$ chance). A go trial (67\% of stop signal task trials, randomly selected) was indicated by a go cue - a peripheral target, whereas a stop trial (33\% of trials, randomly selected) was indicated by an additional appearance of a stop signal - a central gray square (90 pixels square, 2.38 degrees) delayed relative to the go cue presentation with a stop signal delay (SSD). On go trials, subjects were rewarded for a saccade towards the go cue and fixating on it for $200 \mathrm{msec}$; and on stop trials, subjects were rewarded for inhibiting their saccade to go cue, and fixating at the center stop signal for $400 \mathrm{msec}$. Water rewards were provided as feedback, and they were contingent on subject's performance. Rewards were always $125 \mu \mathrm{l}$. The SSD was initialized to a random floating value between 0 and 0.1 secs for every session (day of recording), and they were adjusted in real time with jumps of 16 msecs by a staircase procedure to stabilize at a delay (SSD-50) with 50\% accuracy in the stop trials (Balasubramani et al. 2019). The inter trial interval was 800 msecs (trial baseline).

The choice signal task (Figure 1B) had a similar task framework to stop signal task. We called the choice signal task's counterpart of go trials as accept trials, and that of the stop trials as choice trials. In accept trials (random $67 \%$ of the total choice signal task trials), the subjects made a forced choice to a peripheral offer 1 or the first offer (90 pixels square, 2.38 degrees, positioned at 288 pixels in left or 1632 pixels square, 43.18 degrees, in right of the screen, $50 \%$ chance). In choice trials (random $33 \%$ of the total), an additional second offer (offer 2 or the choice signal) was presented at the center (90 pixels square, 2.38 degrees) delayed with respect to the appearance of offer 1 . The choice signal delay for the presentation of the center offer 2 was the same as the current real time SSD followed for the stop signal task in the session. The offers were associated with either low $(15 \mu \mathrm{l})$, medium $(125 \mu \mathrm{l})$, or high $(250 \mu \mathrm{l})$ reward offer sizes, the rewards were respectively indicated by yellow, blue and magenta colored square cues. In accept trials, the low, medium and high offers were presented with equal probability. Similarly for the center choice signal (offer 2) in choice trials, low, medium and high offers were presented in equal probabilities. In contrast, the peripheral offer 1 in the choice trials was always associated with medium reward size (i.e., blue square); this didn't alter the basic behavioral performance of the subjects in choice trials as a function of value (Figure 21). So, the cue (blue square, peripheral offer 1) that differentially predicted a choice trial, occurred with probability $=0.6$, while probability of a choice trial with non-blue offer 1 was 0 .

The stop signal task and the choice signal task were stochastically counterbalanced. Specifically, the two tasks were randomly alternated with the constraint that each trail repeated from 1 to 3 trials (number chosen in random).

Subjects had never previously been exposed to decision-making tasks in which counterfactual information was available. Previous training history for these subjects 
194 included two types of foraging tasks (see Blanchard et al. 2014; Blanchard and Hayden 195 2015), several gambling tasks (Farashahi et al., 2018; Yoo and Hayden, 2020; Azab and 196 Hayden 2017 and 2018; Heilbronner and Hayden, 2016), attentional tasks (similar to 197 those in Hayden and Gallant 2013), and two types of reward-based decision tasks 198 (Sleezer and Hayden 2016; Wang and Hayden 2017).

\section{Behavioral analysis}

Reaction time was defined as the time taken to saccade to the peripheral target and was computed relative to the presentation time of the go cue or the first offer 1 . In case of a stop trial or a choice trial, the successful cancellation time (or equivalently, the decision time or the choice time in the choice signal task) was the time taken to cancel the saccade by selecting stop signal or the second offer 2, and was computed relative to the presentation of the first offer or the go cue (Schall 1991; Hanes and Schall 1995; Logan et al. 2015).

We used two different methods to compute stop signal reaction time (SSRT). They were 1) median 2) integration methods. We subtracted SSD-50 from the median of go-trial distribution to find stop signal reaction time (SSRT) in the median method. On the other hand, SSD-50 was subtracted from the time point in go-RT distribution whose area was half the distribution total, for computing SSRT in the integration method. We found that SSRT computed through both of the above methods gave nearly equal results, and so, we averaged the values obtained from both methods to obtain final SSRT estimates reported for each subject.

\section{Statistical methods}

Separate PSTH matrices were constructed by aligning spike rasters to the presentation of the go cue or stop signal, appropriately for analysis, for every neuron. Firing rates were calculated in $1 \mathrm{msec}$ bins but were generally analyzed in longer epochs. We normalized by subtracting the mean neuronal firing during inter-trial interval (ITI) time period and z-scored each neuronal data, for bringing them to a common scale of analysis; the normalized data was used for all the decoding analysis mentioned below.

For display, PSTHs were smoothed using 200 msec running boxcars in Figure 4.

\section{Decoding analyses}

The decoding analysis used in this study was similar to our recent publication (Balasubramani et al., 2019). Overall, the procedure involved firstly generating of pseudopopulation activation patterns (see Mante et al. 2013; Stokes et al. 2013) similar to that used for a multi-dimensional scaling method for obtaining OFC ensemble activation patterns (for example: Rigotti et al. 2013; Stokes et al. 2013; Cunningham and Byron 2014; Stokes 2015). Second, we performed binary classifications with the OFC activations (for example: Zhang et al. 2018). To generate population activation states as input patterns for the decoding analysis, we first separated all trials of each neuron by trial conditions. Then, we averaged the activity from randomly sampled 10 trials belonging to a condition for a neuron, with replacement, to form activation states. The 
activation states of all neurons $(\mathrm{N}=96)$ were pooled together to generate a population activation state pattern. 150 unique population activation patterns averaged for some particular time period (either pre-cue or post-cue time periods described below) within the trial were used for decoding analysis.

The shuffling of trials for generating activation states ensured the absence of any temporal relationship between the trials associated with every neuron in the population temporal relationship between neurons, that were representative of "simultaneous" recording. Therefore, it is valid for our method to be applied for ensemble decoding irrespective of its simultaneous recording nature, i.e., both for simultaneous and nonsimultaneous recordings.

The network had a single hidden layer with 100 hidden nodes, and 2 output nodes each representing one target condition for classification. The number of input nodes equal to the total number of neurons used for analysis $=96$ (from two subjects). The network weights were initialized to small random numbers between -0.01 and 0.01 .

The back-propagation algorithm was used for training the decoders (Werbos 1974; Rumelhart et al. 1986; Rumelhart et al. 1988; Haykin and Network 2004). In the below eqn. 1 , the input nodes are denoted by subscript, $k$, hidden nodes by subscript, $j$, and output nodes by subscript, $i$. Output error, $e$, associated with the network's response for the $p$ 'th input pattern is then given by

$$
e_{\mathrm{i}}=\text { desired output }-\mathrm{y}_{\mathrm{i}}
$$

where $y_{\mathrm{i}}$ is the $i$ 'th output node response, and desired output is $1 / 0$ if the $i$ 'th output node is associated with target trial condition for the corresponding input pattern (e.g., successful stopping, failed stopping). Total output error over all input patterns is computed by,

$$
E=\sum_{p} E_{p}
$$

260 Network's objective was to minimize the squared output error for the $p$ 'th pattern as

261 denoted by eqn. (3).

$$
E_{p}=\frac{1}{2} \sum_{i} e_{i}^{2}
$$
A hyperbolic tangent function $(g)$ with slope $=5$ was used as the activation function for every node in the network. The learning rate was set to 0.001 for the network. The node with the maximal activation value in the output layer was taken as the network output response.

We focused our analyses on two key time periods of the trial (1) the $300 \mathrm{~ms}$ epoch before the trial started called the pre-cue epoch, which was actually the fixation time before the presentation of any stimulus targets. (2) The variable time after the go cue (or the first offer in case of the choice signal task) and before the reaction timepoint called the post-cue epoch.

For pre-cue decoder, the training data were population activation states generated by averaging neural activity from the pre-cue epoch of a trial respectively. Similarly, the training data for the post-cue decoder was computed by averaging the post-cue epoch. As mentioned earlier, the pre-cue and post-cue decoders were two class decoders with two output nodes, that distinguished two different trial types. We used the decoders to 
identify various trial types such as successful vs. failed stop trials, go vs. successful stop trials in the stop signal task. In choice signal task, the accept trials with were divided based on non-high (containing the low and medium offer sizes) vs. high offer sizes to classify into two-classes. Accept vs. reject trial types were also analysed as two different classes in the decoders.

The artificial neural network was run for $n=100$ instances with different random weight initializations for obtaining average output performance. Training procedure in all instances converged to classification accuracy of above $80 \%$, and the converged weights at the end of training were used for the testing phase of the decoders. The epoch used for generating activation states for training were from one task and for testing was activation states of the same epoch but from the alternate task. The testing was done on an independent test dataset that was distinct from the one used for fitting. Specifically, we did a $60 \%$ training / $40 \%$ testing split of our population data, and we made sure different trials were used for generating the population training and testing states. We also ensured each training and testing set was stratified- it had equal proportion of different trial types (classes) during training and also testing. We verified whether the decoding performance was significantly higher than $50 \%$ using chi-square test $(\mathrm{p}<0.05)$. Furthermore, we performed permutation tests as a control. In the permutation tests, we shuffled the labels for train and test $5 \mathrm{~K}$ times for generating a null distribution, and we found its 95 percentile value was 80 percent decoding performance. To that end, we present only the significant results with classification percentages greater than 80 (95 percentile null distribution value) in Table 1.

The decoder was sensitive to the temporal activations of each single neuron independently in an ensemble. Our decoder worked on the activations of single neurons, in an independent fashion, and related the ensemble activation state to behavior. That is, the decoder cared about the temporal activity within a neuron i.e., the event locked response activity of every independent single unit. Specifically, we had suggested that the decoding efficiency to be higher for an ensemble activity for precise time periods from the presentation of the stop signal in our recent study (Balasubramani et al., 2019).

This approach of using a multidimensional state decoding of an ensemble with "non-simultaneously" recorded cells was used by multiple other studies (example: Gochin et al. 1994; Thomas et al. 2001; Averbeck et al. 2006). This is a subtler point, but important. Our method is logically equivalent, in many ways, to several other dimensionality reduction approaches that were used for neuronal ensembles (for a review, see (Cunningham and Byron, 2014), and other studies, (Rigotti et al., 2013; Stokes, 2015; Stokes et al., 2013). It is well known that these methods can be done with no problems on asynchronously collected cells, for the same reason that our similar methods can. We had also applied our methods to simultaneously recorded smaller ensembles and found consistent results (Balasubramani et al., 2019)- so our decoding method is general enough to analyse the activation states of neural ensembles either recorded simultaneously or constructed as pseudo-population (Averbeck et al., 2006; Gochin et al., 1994; Thomas et al., 2001). 


\section{RESULTS}

\section{Behavior in the stop signal task and choice signal task}

326 Subjects performed two interleaved tasks, a classic stop signal task (Hanes and

327 Schall, 1995; Figures 1A and Methods), and a novel choice signal task. We reported

328 some of the results on the stop signal task previously (Balasubramani et al, 2019). Our

329 focus here is on comparing those stop-signal results with results in a second task (the

330 choice signal task) that was collected in interleaved trials. Those data were not previously

331 analyzed.

332 The stop signal task consists of two kinds of randomly interleaved trials: go trials

$333(67 \%)$ and stop trials (33\%). On either of these two trial types, following a central

334 fixation, subjects first saw an eccentric target (go cue). On go trials, a juice reward was

335 provided following a successful saccade to the cue. On stop trials, a second signal (stop

336 signal) appeared at central fixation point, and the subjects had to countermand the

337 previously instructed saccade for a reward. Failure to inhibit resulted in no reward. These

338 were called failed stop trials while successful inhibition resulted in a reward and were

339 called successful stop trials. The delay between the go cue and the stop signal is called

340 the stop signal delay (SSD); we used staircase procedure in real time during each session

341 or the day of recording to stabilize at a delay with $50 \%$ success in stopping (SSD-50, see

342 Methods), and computed the stop signal reaction time (SSRT, Logan and Cowan 1984;

343 Logan 1994; Verbruggen and Logan 2008). The SSD-50 was 0.27 sec for subject J and

$3440.15 \mathrm{sec}$ for subject $\mathrm{T}$ and SSRT was $0.14 \mathrm{sec}$ for subject $\mathrm{J}$, and $0.12 \mathrm{sec}$ for subject T

345 (Balasubramani et al, 2019). 
347 isomorphic to the stop signal task. It used interleaved accept trials (66\% of trials),

348 analogous to go trials, in which the subject made a forced choice by accepting a first offer

349 presented peripherally on the screen (offer 1), and choice trials, analogous to the stop

350 trials, where the subjects were presented with not just the peripheral offer but also a

351 second offer (offer 2) at the center. The trials in which the subject chose the offer 1 were

352 called choose offer 1 trials; trials in which the subject chose center offer 2 were called the

353 reject trials or choose offer 2 trials. Reject trials were analogous to successful stop trials

354 (see Methods for details; the second offer was presented 33\% of times as a choice signal

355 with the same delay as the current real time SSD of the stop signal task). In the accept

356 trials, the offers were either low (yellow color cue), medium (blue), or high (magenta) in

357 reward size value (Figure 1B), each presented with equal probability; And in the choice

358 trials, the peripheral offer was always with medium reward size (blue) while the center

359 one was either low, medium or high in offer size, presented with one thirds of probability

360 each. Subjects learned this task well, and successfully chose offers with relatively high

361 reward about $89 \%$ of the times (Supplementary material 1).

A stop signal task

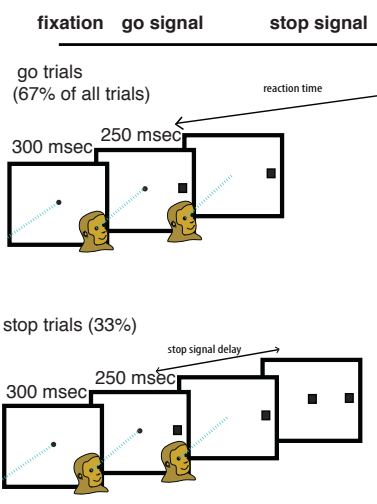

response feedback
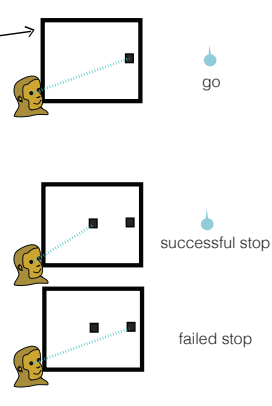

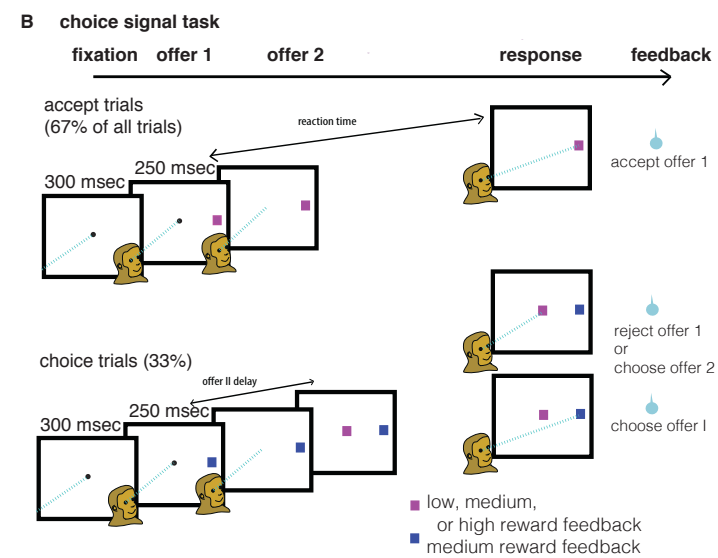

363 Figure 1- Task framework. (A) stop signal task showing go trials, successful and failed stop

364 trials (B) choice signal task showing accept, choose offer 1 and choose offer 2 (reject) trials. 
367 metrics between the stop signal task and the choice signal task for both the subjects $\mathrm{J}$ and

368 T appear in Figure 2. Reaction times on accept trials (choice signal task) correlated with

369 the go trial reaction times (stop signal task) significantly across sessions (subject J:

370 Pearson correlation, $\mathrm{r}=0.90, \mathrm{p}<0.001$; subject $\mathrm{T}$ : $\mathrm{r}=0.86, \mathrm{p}<0.001)$. That means day to

371 day variability in performance affected the two tasks in a similar way. Likewise, reaction

372 times on choose offer 1 trials were correlated with those on the failed stop trials (subject

373 J: Pearson correlation, $r=0.90, p<0.001$; subject $\mathrm{T}: \mathrm{r}=0.72, \mathrm{p}=0.005)$. Choice

374 accuracy (selecting an offer with relatively larger reward size) when found with respect

375 to the delay in the presentation of offer 2 (i.e., SSD) significantly correlated between

376 subjects, particularly, as the delay between the presentations of offer 1 and offer 2

377 increased, the accuracy in choosing the best of the offers decreased (Figures $\mathbf{2 E}$ and $\mathbf{2 F}$;

378 Supplementary material 1 ). A similar behavior was seen in the stop signal task, where

379 there was a decrease in the percentage of successful saccade inhibition (i.e., inhibition

380 accuracy) with increase in SSD (Figures 2E and 2F). Note that subject T had higher

381 variance in his inhibition function during longer delays but the behavior was still

382 monotonic, allowing us to study his neural and behavioral data as a function of delay

383 time, particularly around the SSD-50 and SSRT periods (Figure 2F); these longer delay

384 trials did not affect the interpretation and results of our study, and our neural decoding

385 analyses described in this study do not focus on the effects of SSD on behavior. 

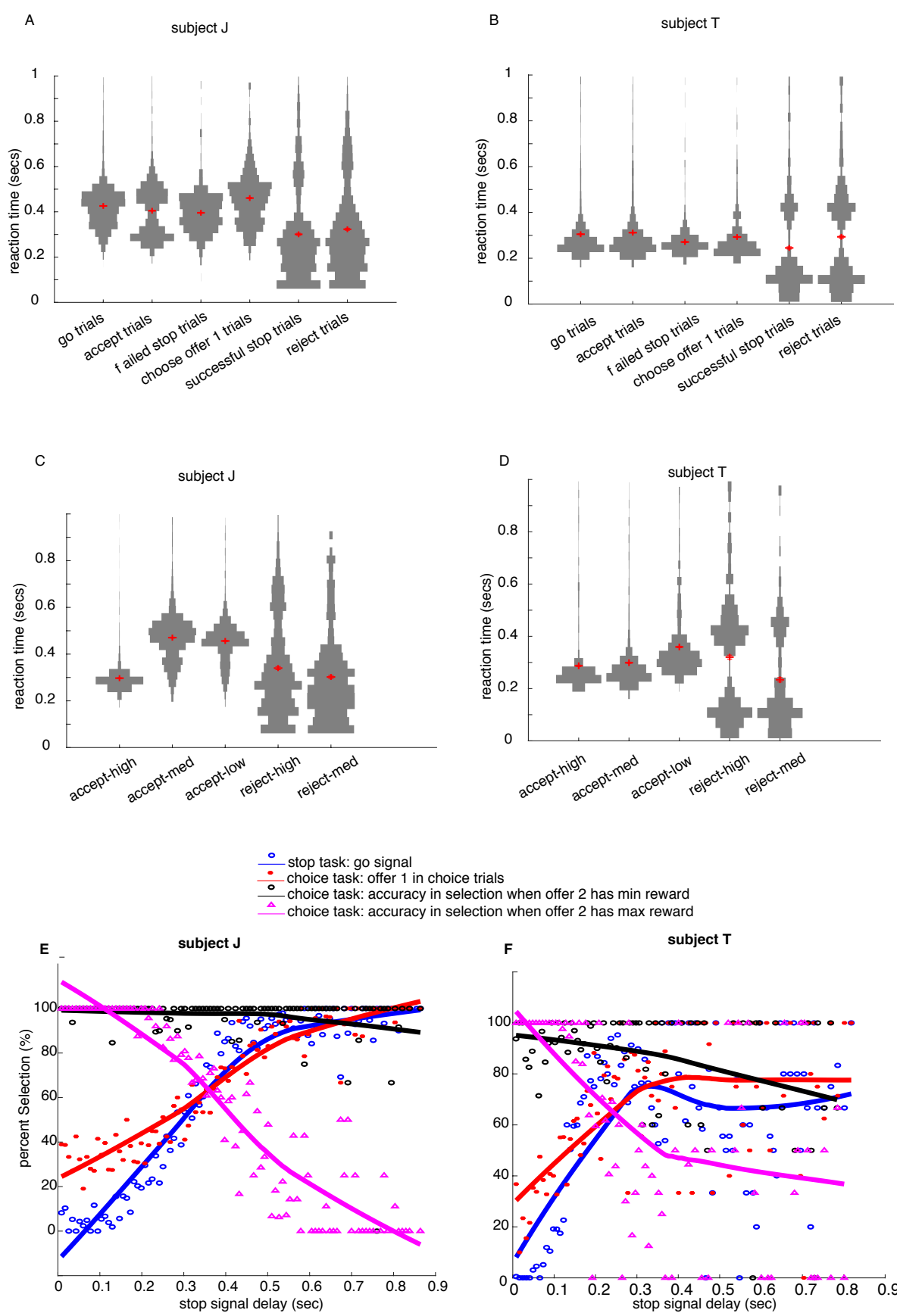

ection when offer 2 has min reward

Figure 2: Behavioral results. For subject $J$, the results are presented in panels $(A, C, E)$ and for subject $T$ in $(B, D, F)(A, B)$ reaction time violin distributions for various trial conditions of stop signal task. The red centroids in the distribution plots denote the mean and the $+/$-standard error of mean. An extended data representation (Supplementary material 2) shows the cumulative histogram as a function of reaction times for various trial types. (C, D) reaction time distributions for various trial conditions of choice signal task. Another extended data (Supplementary material 3) shows the session-wise SSD distributions, and SSRT distributions of both subjects, J and T. (E) Inhibition function and accuracy of choices (choosing the best of offers) varied as a function of SSDs. The inhibition function for both the subjects showed sensitivity to the magnitude of stop signal delay (shown in blue lines). ( $F$ ) The choice functions for different offer reward sizes are shown. The data points in red indicate the percent selection of offer 1 (medium reward size) 
in choice trials. This varied similar to the inhibition function. The data points in black indicates the accuracy of choice when offer 2 had minimum offer size, and that accuracy was larger compared to another case where offer 2 had maximum reward size (magenta). This suggests that the subjects were relatively more greedy in choosing larger offer sizes $(125 \mu \mathrm{l}, 150 \mu \mathrm{l})$ especially when contrasted with an offer with minimum reward size $(15 \mu \mathrm{l})$. The continuous lines in inhibition and choice functions are shape preserving interpolants of the scattered data points obtained by data

407 support for the hypothesis that subjects treat these tasks in analogous ways (Figure 3).

408 Further supporting this idea, the reaction times of the go trials that followed a successful

409 stop trial were correlated with the reaction times of the accept trials that followed a reject

410 trial in the choice signal task (Pearson correlation, $r=0.71, \mathrm{p}<0.001$ ). Likewise, we saw

411 a significant correlation between the reaction times of saccade trials that followed another

412 saccade trial in both the tasks (go trials / accept trials; $r=0.88, \mathrm{p}<0.001$ ).
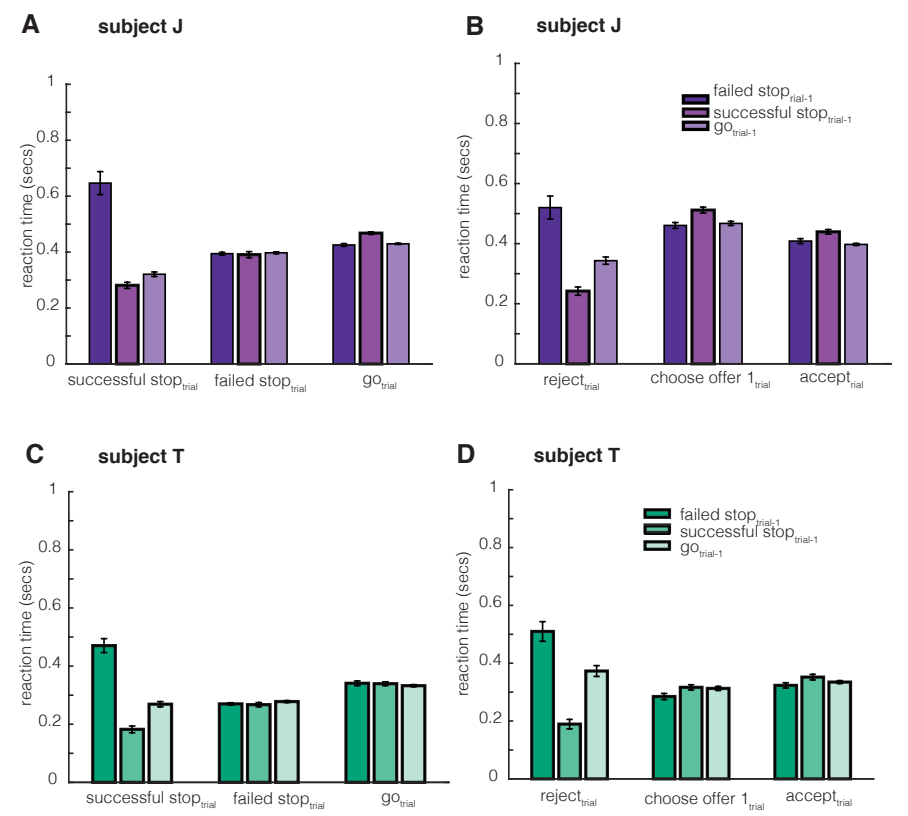

414 Figure 3: Trial-to-trial behavior effects. For various trial conditions of stop and choice signal ask- we found trial history based effects in reaction time behavior in (A, C) stop signal task and $(B, D)$ choice signal task. Error bars represent SEM. Accept trials were generally longer when followed an reject trials in contrast to following another accept trial (subject $\mathrm{J}$ figure 3B: $0.05 \mathrm{~s}$, tstat $=3.41, p<0.001$; subject T figure 3D: tstat $=2.06, p=0.039$ ). Similar effects were found for stop signal task: Successful stop trials were shorter when after another successful stop in 
420 contrast to following a failed stop trial (subject $\mathrm{J}$ figure $3 \mathrm{~A}: 0.36 \mathrm{~s}$, t-stat $=11.33, \mathrm{p}<0.001$;

421 subject T figure $3 \mathrm{C}: 0.29 \mathrm{~s}$, t-stat $=11.88, \mathrm{p}<0.001)$.

422

\section{Stopping and choice at the level of single neurons}

424 We recorded responses of 96 neurons (52 in subject $\mathrm{J}$ and 44 in subject $\mathrm{T}$ ) in Area

42513 of the OFC (see Balasubramani et al, 2019). Note that while this number of neurons is

426 smaller than in some other studies, it is sufficient to have detected the effects we discuss

427 and thus suits our purposes. We show responses of three example neurons in Figure 4.

428 As is typical in OFC, neurons had diverse tuning (coding) profiles. 
bioRxiv preprint doi: https://doi.org/10.1101/2020.04.23.057455; this version posted April 25, 2020. The copyright holder for this preprint (which was not certified by peer review) is the author/funder, who has granted bioRxiv a license to display the preprint in perpetuity. It is made available under aCC-BY-NC-ND 4.0 International license.
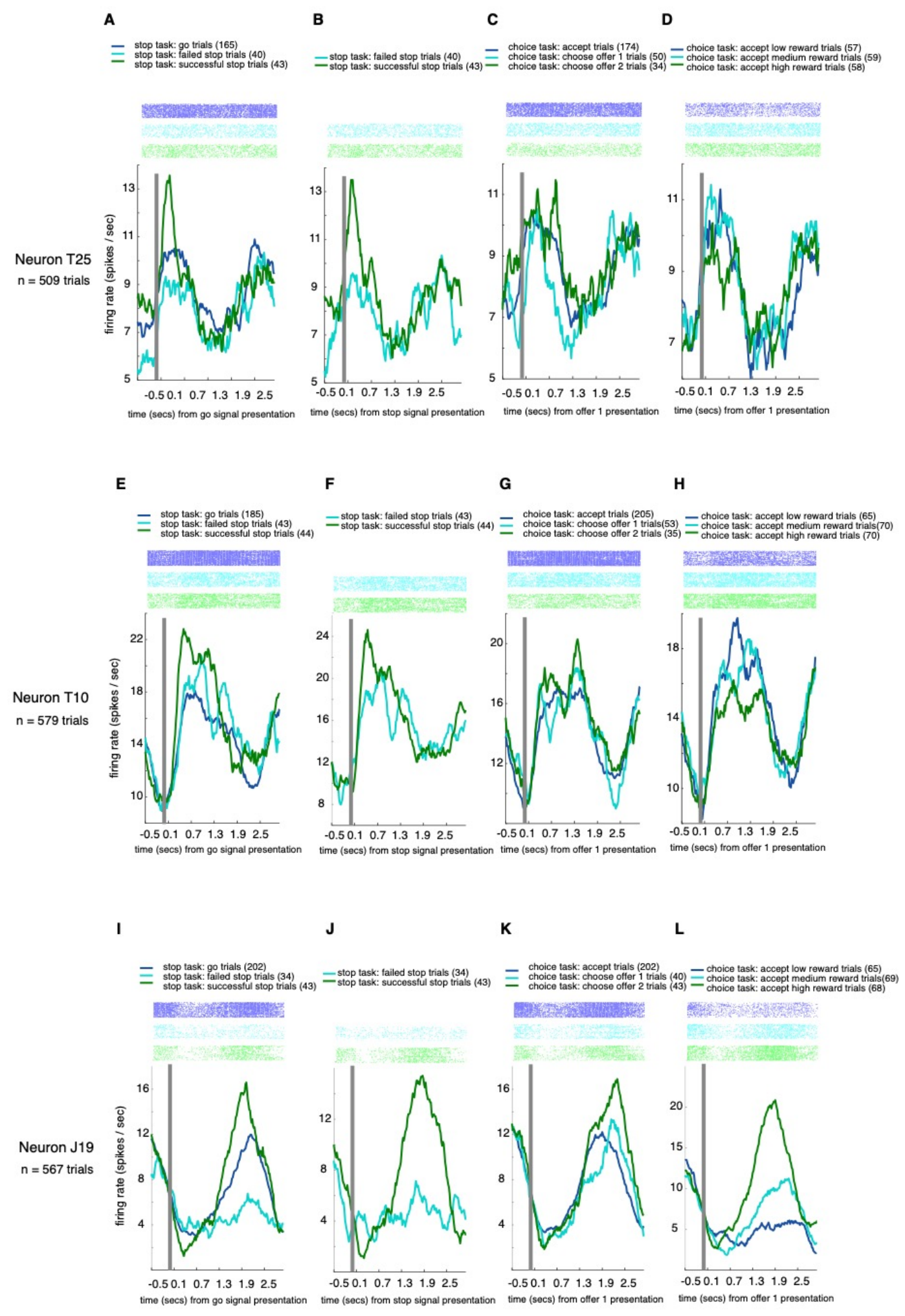

430 Figure 4: Illustration of sample neurons. We present neuron T25 in panels A-D, neuron T10 in 431 panels $\mathrm{E}-\mathrm{H}$, and neuron $\mathrm{J} 19$ in panel I-L. The first panel of every neuron shows the activity in stop 432 signal task when aligned to the go cue $(A, E, I)$, and that when aligned to the stop signal in the 
second panel $(B, F, J)$. When aligned to the go signal, we show the go, successful and failed stop trials' mean neural activity. And when aligned to the stop signal, we present the mean neural activities for the successful and failed stop trials. The last two panels for every neuron show the matching trials from choice signal task. The panels $(C, G, K)$ present the accept, choose offer 1 and choose offer 2 (reject) trials of each neuron, while the panels $(D, H, L)$ present the accept trials of choice signal task with different offer sizes (high, medium and low offer sizes) of each neuron. Some significant differences in neural firing rates between trial types were seen in the stop signal task: Neuron T25 differentiated successful and failed stop trials in pre-go cue and post-go cue time periods (Figure 4A; pre-cue: ttest, $p=0.01$, post-cue: $p=0.02$ ). The inhibition code could be found even with respect to the presentation of the stop signal, the neuron differentiated the successful and failed stop trials in the post-stop signal time period before the stop signal reaction time $(p=0.01$, Figure 4B). Another neuron, T10, differentiated the successful and failed stop trials in the post-go cue epoch, (ttest, $p=0.04$, Figure $4 \mathrm{E}$ ) and similarly, it also differentiated go vs. successful stop trials ( $p=0.002$, Figure $4 E)$ in the same post-go cue epoch. Neuron $\mathrm{J} 19$ differentiated successful and failed stop trials even before stop signal reaction time in the post-go cue epoch (t-test, $p=0.05$, Figure $4 \mathrm{l}$ ), as well as in the post-stop signal time period before stop signal reaction time $(p=0.019$, figure $4 \mathrm{~J})$. In the choice signal task based trials (Figure 4C), the neuron T25 differentiated choose offer 1 and choose offer 2 (reject) trials in the post-offer 2 presentation time period before SSRT ( $p=0.04$, figure $4 C$ ). The neuron T10's and 452 J19's mean firing activity in the accept trials were negatively correlated to the size of offers during feedback (Pearson correlation, $r=-0.16, p=0.017$ for T10; $r=0.40, p<0.001$ for J19).

456 focused our analyses on comparing stopping and choice processes only in two key time

457 periods of the trial: (1) pre-cue epoch: the $300 \mathrm{~ms}$ epoch before the trial started, which is

458 related to the fixation time before the appearance of any stimulus targets and that can

459 inform about proactive signaling. (2) post-cue epoch: the variable time after the go signal

460 (first offer 1 in choice signal task) but before the reaction time that can inform about

461 reactive signaling.

463 response compared to the baseline, and 51.04\% showed negative trial related response,

464 and the rest weren't significantly modulated (t-test on firing rates in post-cue epoch,

$465 \alpha=0.05)$. We saw a similar pattern in the stop signal task: In $44.79 \%$ of cells, the average

466 firing was higher during the trial compared to baseline and 53.12\% had responded with a

467 negative average firing to the baseline, the rest (2.09\%) showed no change. 


\section{Distinct neural activities for actions and rewards in the two tasks}

Our key question is how firing rate patterns in the two tasks relate to each other.

471 Focusing on the post-cue epoch, and we first asked whether the patterns for go / stop

472 actions in the stop signal task have any similarity to the accept / reject choice patterns. In

473 other words, does going in the former task correspond - neutrally - to accepting in the

474 latter task? This pattern is expected because the actions are matched. We regressed the

475 firing rate against the action variable (saccade or inhibition of saccade) in the two trial

476 types; coefficients were significantly correlated $(r=0.28, p<0.001)$. This, positive

477 correlation indicates the neurons encode action information in a similar fashion in the

478 same direction. We also correlated unsigned coefficients; a positive correlation is

479 evidence that the two relevant neural populations overlap more than chance (Azab and

480 Hayden, 2017). Here we found a positive correlation as well, indicating that the same

481 groups of neurons are involved in the two tasks $(\mathrm{r}=0.65, \mathrm{p}<0.001)$. Neither result is

482 particularly surprising, although they provide valuable confirmatory evidence and set the

483 stage for our subsequent results.

484 Next, we asked how neural patterns for the two tasks relate in terms of reward

485 magnitudes associated with chosen options (also called the value of chosen option). In the

486 stop signal task when the stop signal is presented, only successful stop trials provided

487 rewards, that means the action and reward magnitude identifiers were one and the same

488 in stop trials and they cannot be used differentially identify reward magnitude patterns.

489 Moreover, because of this confound, any similar effects between stop signal task and

490 choice signal task patterns may reflect their shared actions (and OFC has spatial tuning,

491 Feierstein et al. 2006; Roesch et al. 2006; Strait et al. 2016; Yoo et al. 2018) instead of 
492 chosen option values. In order to understand the reward value effects, we controlled for

493 the trials in the choice signal task. We used only accept trials, thus the action was the

494 same - a saccade, in the choice signal task. We regressed the neural activity in the post-

495 cue epoch and the actions (saccade or inhibition of saccade) in the stop trials, and, in

496 parallel, we regressed the post-cue neural activity with the received reward sizes only in

497 the accept trials. We then asked whether the regression coefficients from two tasks were

498 significantly related. If they were related, it would imply OFC had similar codes for

499 action value and reward value. Interestingly, the regression coefficients computed for

500 each neuron in two different tasks weren't related for the post-cue epoch (Pearson

501 correlation, $\mathrm{r}=0.06, \mathrm{p}=0.58$ ). Moreover, the unsigned coefficients weren't correlated

502 (Pearson correlation, $\mathrm{r}=0.10, \mathrm{p}=0.31$ ). This implies OFC neuronal codes for stopping

503 and choice value don't have a simple linear relationship in the post-cue epoch.

504 In a previous study, we had found that subjects perform reward value

505 comparisons between offers for making their choice (Strait et al., 2014). Since go / stop is

506 associated with a distinct outcome in stop trials, we next asked whether the principles

507 regulating choice can be applied to stopping, that is performing reward value comparison

508 between actions. To answer this question, in the choice signal task, we estimated how the

509 neural activity during choice was related to reward value differences between offers.

510 Specifically, we regressed the neural firing in the post-cue epoch of choice trials with

511 their offer size differences (offer 2 - offer 1). And in the stop signal task, to estimate how

512 the neural responses were related to actions, we regressed the firing activity in the post-

513 cue epoch of the stop trials with the action regressor (saccade or inhibition of saccade).

514 We compared the computed coefficients for each neuron, and we didn't find any 
515 significant relationship (Pearson correlation between the signed coefficients: $r=0$,

$516 \mathrm{p}=0.95)$, but of significance was the relationship between the unsigned coefficients $(\mathrm{r}=$

$5170.52, \mathrm{p}<0.001)$. This result with the unsigned coefficients suggests that an overlapping

518 subset of neurons assists both choice and stopping in the OFC, although their codes aren't

519 relatable in a simple and linear fashion as implied by the signed coefficients.

521 Overlapping functional ensemble codes for stopping and choice

522 In the earlier section, we saw action related and reward related relationships at the

523 level of firing rates between the tasks. Firing rates for actions were highly correlated

524 between tasks, and these codes weren't simply related to the reward signals. We then

525 wanted to examine the extent of overlap between the tasks at the level of population

526 ensembles. Notably, population averages revealed no significant differences between

527 stopping / going trial types in the stop signal task as well as reject / accept trial types in

528 choice signal task (Figure 5). 
subject $\mathbf{J}$

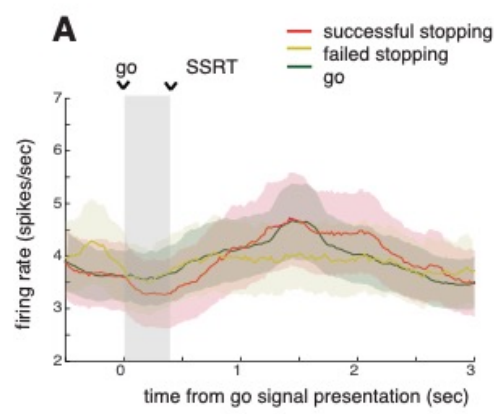

B
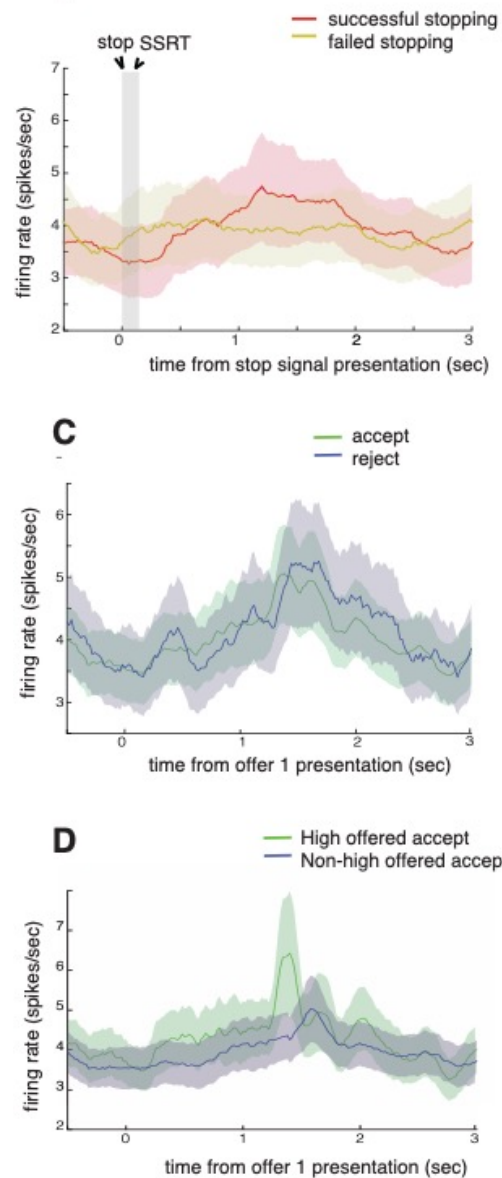

subject $\mathrm{T}$

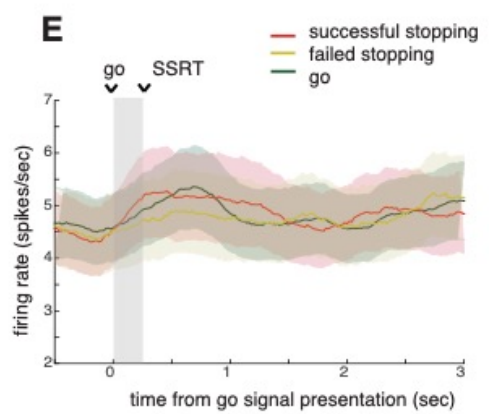

$\mathbf{F}$
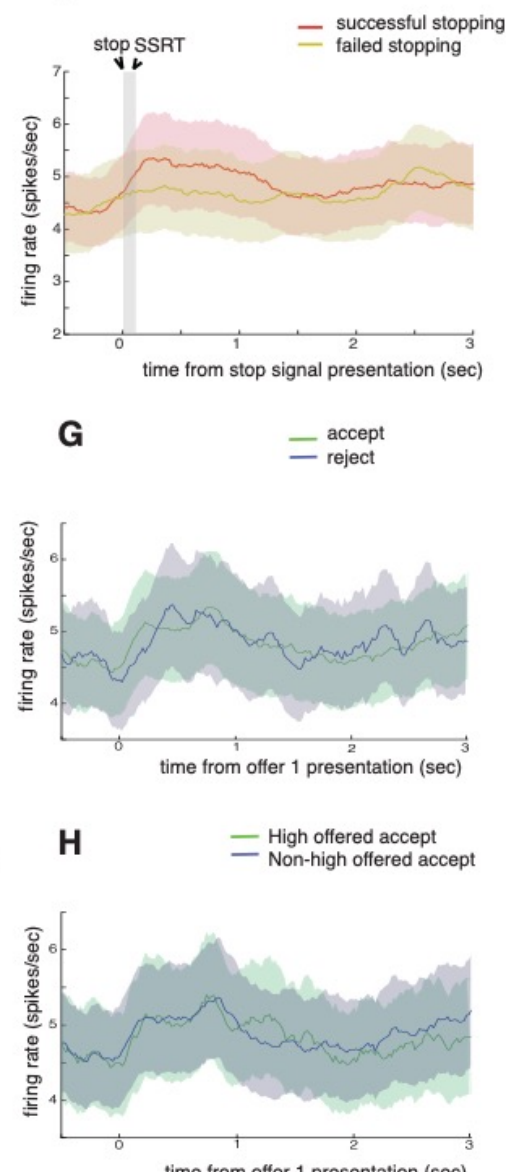

time from offer 1 presentation (sec)

$\begin{array}{ll}530 & \text { Figure 5: population averages provide weak information about stopping and rewards. } \\ 531 & \text { Population activity for successful stopping and failed stopping with respect to (A, E) go cue }\end{array}$ 532 presentation and (B, F) stop signal presentation, for subjects $\mathrm{J}$ and T. Time from start of the go 533 cue to SSRT is shaded in panels A and E (and stop signal presentation to SSRT in $B$ and $F$ ).

534 Data for all SSDs are averaged to present the signals during successful and failed stop trials.

535 They didn't reveal significant information about the pattern of stopping. In the same lines, 536 population responses for accept and reject trials for choice task in subjects $\mathrm{J}$ and $\mathrm{T}$, respectively, 537 are shown in (C, G); they didn't reveal significant information about choice patterns. Finally, the 538 population averages for high and non-high offering accept trials are presented in $(\mathbf{D}, \mathbf{H})$ for 539 subjects $\mathrm{J}$ and $\mathrm{T}$, respectively. Error bars in all panels denote SEM. 
542 information that may not be conveyed at the level of single units (Zemel et al. 1998;

543 Averbeck et al. 2006b; Meyers et al. 2008; Morcos et al. 2018). We therefore made use of

544 neural network decoders, which provide the most direct way to detect patterns in the

545 ensemble with minimum number of assumptions. This approach has been used

546 successfully in several other studies (for example: Rigotti et al. 2013; Stokes et al. 2013;

547 Cunningham and Byron 2014; Stokes 2015). We trained two different decoders, first one

548 was trained on stop signal task and tested on choice signal task; the second one was the

549 reverse (and was used for confirmation). These analyses used the same two epochs as

550 above (Table 1). In any instance, population activation patterns $(\mathrm{N}=96)$ were generated

551 (see Methods) from only one of the epochs (pre-cue, post-cue) for both training and

552 testing, and they were fed in as input to the decoder. In case of shared neural patterns

553 underlying stopping and choice, a decoder trained on just one of tasks would perform

554 with high efficiency when tested on the other task (the results should survive statistical

555 corrections for significance: chi-square test, $\mathrm{p}<0.05, \geq 95$ percentile threshold in

556 permutation test, see Methods). Our results below indeed suggest the existence of

557 overlapping ensemble patterns between stopping and choice.

558 We examined the decoding performance of some key patterns or trial differences

559 in stopping and choice: (1) successful vs. failed stop trials, where the trials not just

560 differed in actions (only successful stop trials were associated with inhibition of saccade)

561 but also differed in reward sizes (no reward during failed stop). (2) go trials vs. failed

562 stop trials, where the trials only differed in reward sizes (only go trials were associated

563 with reward) but not in actions (saccade). Their counterparts in choice trials: (3) accept 
564 vs reject trials, where the trials differed both in actions (only reject trials were associated

565 with inhibition of saccade) and in offer values. (4) high vs. non-high offering accept trials

566 of the choice signal task, where the trials only differed in reward sizes but not in actions

567 (saccade). The results (Table 1) show that the decoders trained to distinguish the trials

568 belonging to one task but tested on the trials belonging to the other, performed with

569 greater than $80 \%$ efficiency, indicating strong overlap between stopping and choice

570 ensemble codes, both in the pre-cue epoch and post-cue epoch. Particularly, the decoders

571 trained on choice signal task activations had over $97 \%$ efficiency in decoding the

572 successful vs failed trials of the stop signal task (Table 1, highlighted in yellow),

573 suggesting a relatively stronger overlap of stopping to choice than the vice versa.

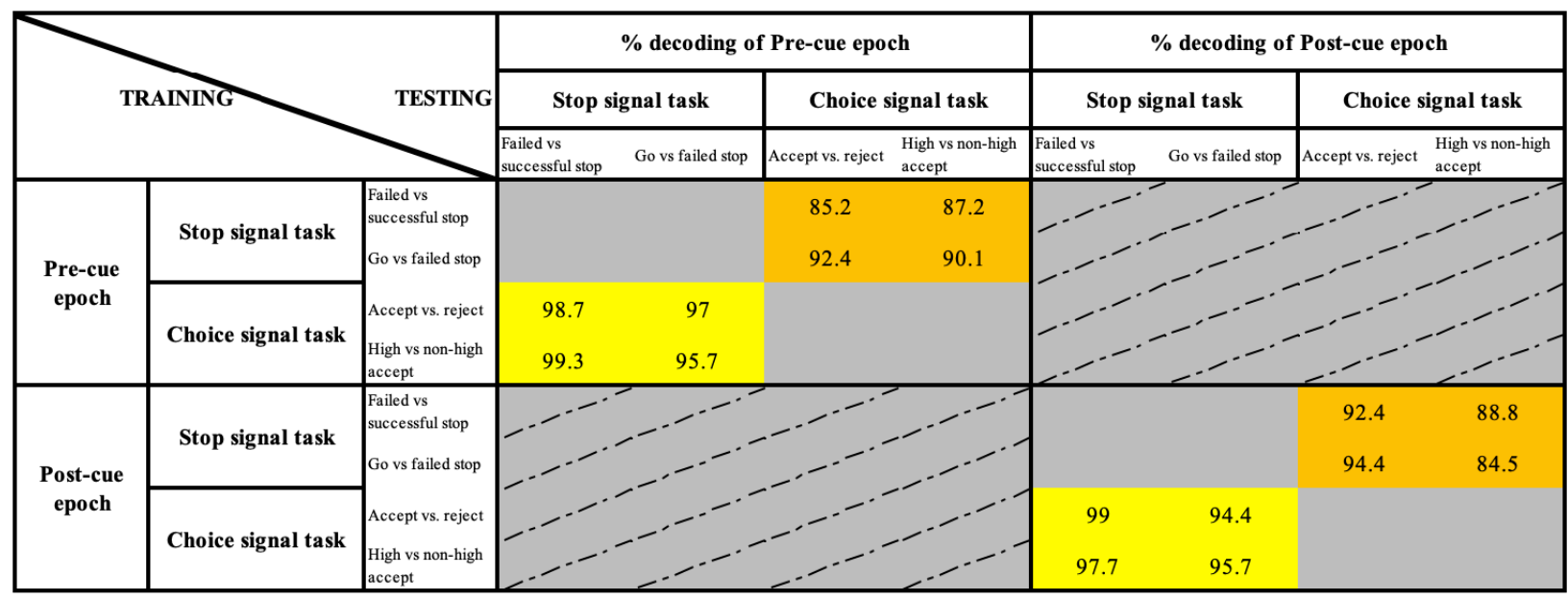

trained in a choice signal task but tested in stop signal task 
584 Reward promotes choice of the option it is associated with. The results show that

585 OFC ensembles make use of similar patterns for stopping and offer value (Hayden 2018),

586 and in terms of direction of the effect, ensemble patterns for stopping are more similar to

587 choice patterns. Similarities between neural patterns of both the tasks may promote the

588 idea of shared strategies between stopping and choice related decisions. 


\section{DISCUSSION}

591 We examined responses of neurons in area 13 of the OFC in two tasks, one an

592 implementation of a classic stop signal task and the other a simple choice signal task with

593 similar structure. Although the economic role of this region in choice is well established,

594 its role in stopping is not. Our earlier finding that OFC ensembles predict inhibition both

595 before the trial and immediately before the stopping response suggest that this region

596 does participate in regulation of stopping (Balasubramani et al., 2019). The timing of the

597 two stopping-related patterns is reminiscent of the times associated with proactive and

598 reactive control, respectively (Hanes et al. 1998; Stuphorn et al. 2000; Ito et al. 2003;

599 Braver et al. 2007; Chikazoe et al. 2009; Chen et al. 2010; Stuphorn et al. 2010; Braver

600 2012; Stuphorn and Emeric 2012; Majid et al. 2013). In the present study, by interleaving

601 the tasks for stopping and choice, we show, first, that it is largely the same neurons

602 participating in both processes. Second, through population analysis and pattern

603 decoders, we show the ensemble patterns that differentiate value of offers underlying the

604 choice signal task can distinguish failed from successful stopping. These results thus

605 support the hypothesis that stopping and choice signal reflect common computations

606 occurring in overlapping circuits.

607 There is some reason to think that stopping and choice may spring from shared

608 processes. For example, several psychiatric conditions, including depression and

609 addiction, impair both processes, and greater impairment of both is associated with

610 greater disease progression (Nestler et al. 2002; Iacono et al. 2008; Volkow et al. 2011).

611 Second, both are closely associated with, and empirically linked to, the broader concept

612 of self-control (Inzlicht et al. 2014; Berkman et al. 2016; Shenhav 2017). Earlier studies 
613 have referred to both inhibitory control and choice processes to reflect upon the nature of

614 "self-control". Some examples include controlling of choice towards a tempting sub-

615 optimal option (e.g. Baumeister and Newman 1994; Mischel et al. 1996; Muraven and

616 Baumeister 2000; Shenhav et al. 2013; Shenhav 2017), and it extends to even avoiding

617 the sub-optimal ones (Fujita 2011; Berkman et al. 2016). One theory of self-control sees

618 it as a result of competition between two systems- impulsive (hot) and controlled (cold)

619 systems (James 1890; Baumeister and Heatherton 1996; Carver 2005) this relates to the

620 view of inhibitory control (that studies the ability to successfully inhibit a response

621 process) as a form of self-control—where a cold system tries to win over the hot one. On

622 the other hand, many studies suggest the view of self-control as an economic decision,

623 i.e., a comparison of two different utility options (McGuire and Kable 2015; Berkman et

624 al. 2016).

625 One of the major motivations for this study was to test a set of hypotheses that

626 have emerged from our laboratory in recent years (Hayden 2018; Hayden and Moreno-

627 Bote 2018; Yoo and Hayden, 2018). These ideas, which we sometimes call the foraging

628 view of economic choice, are inspired by, and somewhat similar to, earlier models of

629 choice, especially work of Krajbich and colleagues and Kacelnik and colleagues

630 (Krajbich et al. 2010; Kacelnik et al. 2011). In foraging theory, decisions are generally

631 framed as accept-reject (Stephens and Krebs 1986; Stephens and Anderson 2001;

632 Kacelnik et al. 2011; Blanchard and Hayden 2015). In our view, attention focuses on one

633 option at a time, the subject evaluates the attended option relative to the value of

634 rejection, and then the subject either accepts or rejects it. In a two-option choice, the first

635 attended option has a high value of rejection, since the cost to inspect the second is very 
636 low, and rejecting the first does not preclude its choice a few hundred milliseconds later.

637 In this view, comparison is, generally speaking, an emergent process. That is, attending to

638 each option triggers a slow evaluation process, one whose speed is determined in part of

639 the value (or strength of evidence) of the attended option (Krajbich et al. 2010). The

640 comparison occurs in a de facto manner, because the stronger option is more likely to

641 reach the threshold sooner. In practice, this approach has many similarities with

642 traditional accept-reject decisions. However, the key differences include asynchronous

643 consideration of options and a change in how choice occurs in single and multiple (more

644 than two) option cases. Specifically, an important difference comes from the fact that

645 choice process involves steps in which the subject chooses between selecting and

646 rejecting the attended option - between going and stopping. Our results provide neural

647 evidence consistent with this idea.

648 These results obtained from our overlapping task design provides evidence in

649 favor of the hypothesis that the neural processes that regulate stopping overlap with the

650 processes that regulate economic choice. One limitation of network decoders used in the

651 study is they do not provide any ready insight into the mechanisms of how neuronal

652 outputs are combined to drive action. Doing so is not our goal here. Instead, our main

653 goal is to understand how neural patterns relate across the two categories of tasks.

654 Overall, our results suggest one core function of OFC may be to generate an

655 abstract regulatory signal to feed into a cascade of downstream structures that ultimately

656 determine choice (Hunt and Hayden 2017; Balasubramani et al. 2019; Yoo and Hayden,

657 2018). In this way, it may be similar to other regions, especially cingulate cortex but also

658 striatal regions (Hillman and Bilkey 2010; Shenhav et al. 2013; Sleezer et al., 2017; 
659 Sleezer et al., 2016). In the context of economic choice, this signal will resemble a value

660 signal; in other cases, it will correlate with other relevant task variables. This view is

661 consistent with the idea that choice and control processes both reflect a gradual

662 transformation of network processes across brain regions obeying principles of

663 distributed systems (Hunt and Hayden 2017; Eisenreich et al. 2017; Balasubramani et al.

664 2018). One benefit of view is that it provides a basis for the observed role of OFC and

665 their adjacent structures in self-control (McClure et al. 2004; Kable and Glimcher 2007).

666 


\section{Supplementary material 1:}

\begin{tabular}{|c|c|c|c|}
\hline & Trial type & \multicolumn{2}{|c|}{$\begin{array}{l}\text { Reaction time distribution medians } \\
\text { (s) }\end{array}$} \\
\hline & & subject $J$ & subject $T$ \\
\hline \multirow[t]{3}{*}{ Stop signal task } & Go trials & $0.43 \pm 0.09$ & $0.27 \pm 0.12$ \\
\hline & Failed stop trials & $0.39 \pm 0.09$ & $0.25 \pm 0.08$ \\
\hline & Successful stop trials & $0.25 \pm 0.20$ & $0.15 \pm 0.22$ \\
\hline \multirow[t]{3}{*}{ Choice signal task } & Accept trials & $0.41 \pm 0.12$ & $0.27 \pm 0.13$ \\
\hline & Choose offer 1 trials & $0.46 \pm 0.12$ & $0.25 \pm 0.10$ \\
\hline & Choose offer 2 (reject) trials & $0.28 \pm 0.19$ & $0.20 \pm 0.23$ \\
\hline
\end{tabular}

\begin{tabular}{|c|c|c|c|}
\hline \multicolumn{2}{|c|}{ Choice signal task } & subject $J$ & subject $T$ \\
\hline $\begin{array}{c}\text { Reaction times versus value } \\
\text { of offers }\end{array}$ & $\begin{array}{c}\text { Forced choice } \\
\text { trials- offer 1 }\end{array}$ & $\begin{array}{c}\text { Pearson correlation, } \mathrm{r}=0.52, \\
\mathrm{p}<0.001\end{array}$ & $\mathrm{r}=0.43, \mathrm{p}<0.001$ \\
\cline { 2 - 4 } & $\begin{array}{c}\text { Choice trials }- \\
\text { offer 2 }\end{array}$ & $\mathrm{r}=0.29, \mathrm{p}=0.003$ & $\mathrm{r}=0.27, \mathrm{p}<0.001$ \\
\hline
\end{tabular}


bioRxiv preprint doi: https://doi.org/10.1101/2020.04.23.057455; this version posted April 25, 2020. The copyright holder for this preprint (which was not certified by peer review) is the author/funder, who has granted bioRxiv a license to display the preprint in perpetuity. It is made available under aCC-BY-NC-ND 4.0 International license.

\begin{tabular}{|c|c|c|c|}
\hline $\begin{array}{c}\text { Preference for offer 1 than } \\
\text { offer 2 }\end{array}$ & Choice trials & $\begin{array}{c}57.6 \% \\
\text { (chi-square test, chi-square }= \\
5.78, \mathrm{p}=0.016)\end{array}$ & $\begin{array}{c}56.25 \% \\
\text { (chi-square stat }= \\
3.38, \mathrm{p}=0.066)\end{array}$ \\
\hline $\begin{array}{c}\text { Preference for offer 1 versus } \\
\text { stop signal delay }\end{array}$ & Choice trials & $\begin{array}{c}\text { slope of psychometric } \\
\text { sigmoidal fit }=5.53\end{array}$ & Slope $=6.93$ \\
\hline $\begin{array}{c}\text { Accuracy (choosing best } \\
\text { offer) }\end{array}$ & Choice trials & $89.25 \%$ & $89.8 \%$ \\
\hline $\begin{array}{c}\text { Accuracy versus stop signal } \\
\text { delay }\end{array}$ & Choice trials & $\begin{array}{c}\text { slope of psychometric } \\
\text { sigmoidal fit = -4.93 }\end{array}$ & Slope $=-5.3$ \\
\hline \begin{tabular}{c} 
Neuronal coding for rewards \\
\cline { 2 - 4 }
\end{tabular} & Offer 1 & $21.15 \%$ & $9.09 \%$ \\
\cline { 2 - 4 } & Offer 2 & $11.5 \%$ & $9.09 \%$ \\
\hline
\end{tabular}

673

674

675

676

677

678

679

\section{Supplementary material 2:}

The reaction time distributions are accumulated in a cumulative plot to show the differences between trial types for both tasks in subjects $\mathrm{J}$ and $\mathrm{T}$.

A

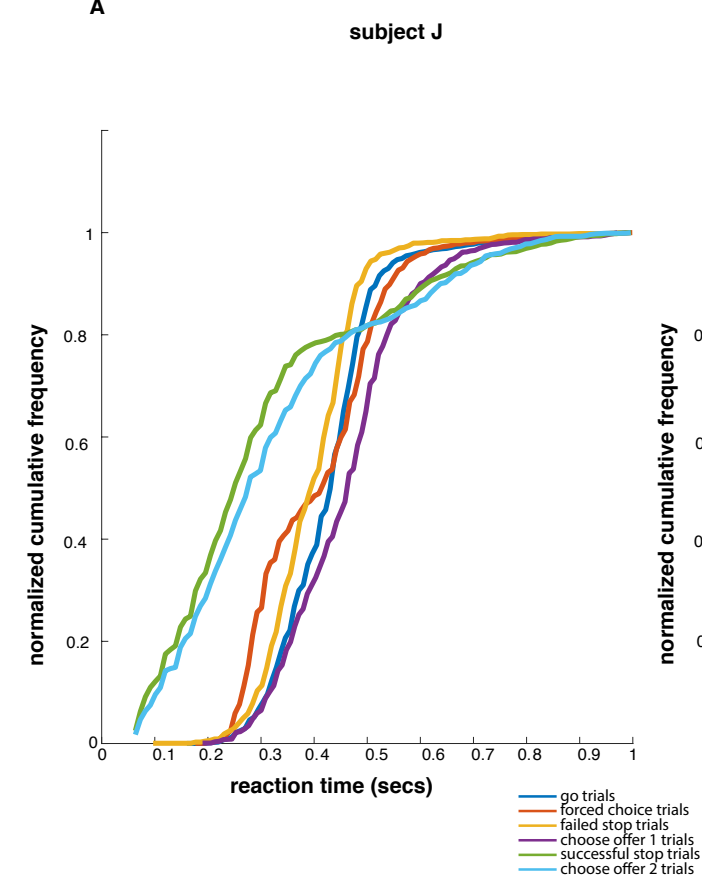

B

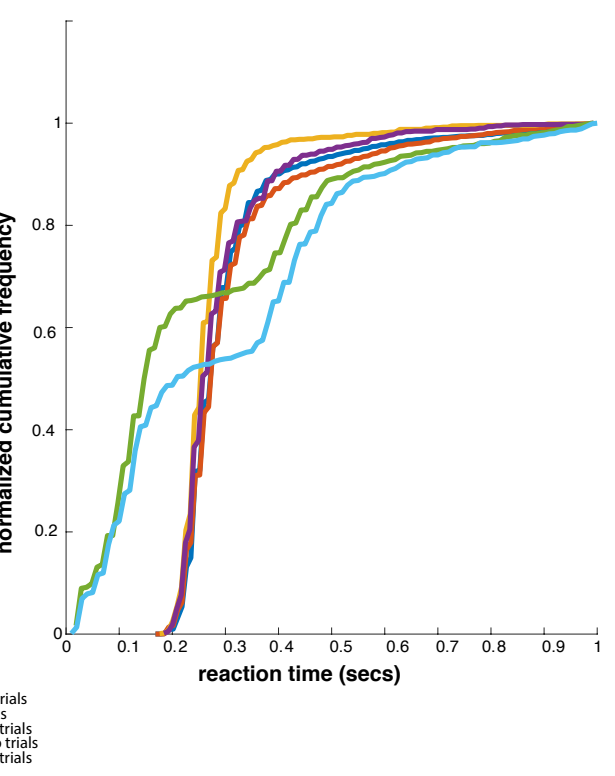




\section{Supplementary material 3:}

683

684

685

686

687

688

689

690

691

692

693

694

695

696

697

698

\section{subject $\mathrm{T}$}
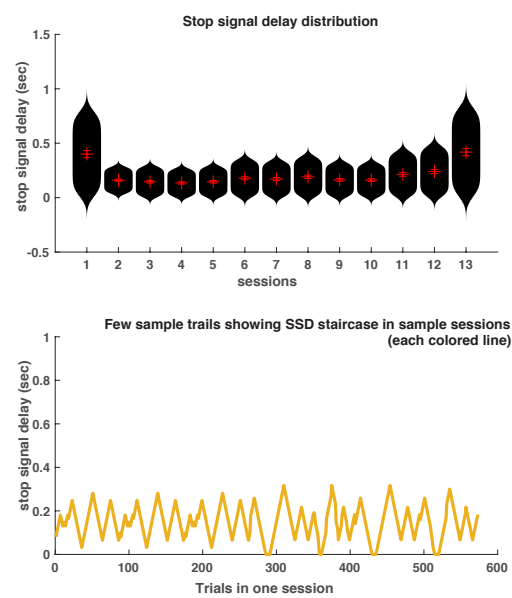
subject $\mathrm{T}$.

\section{subject $\mathbf{J}$}
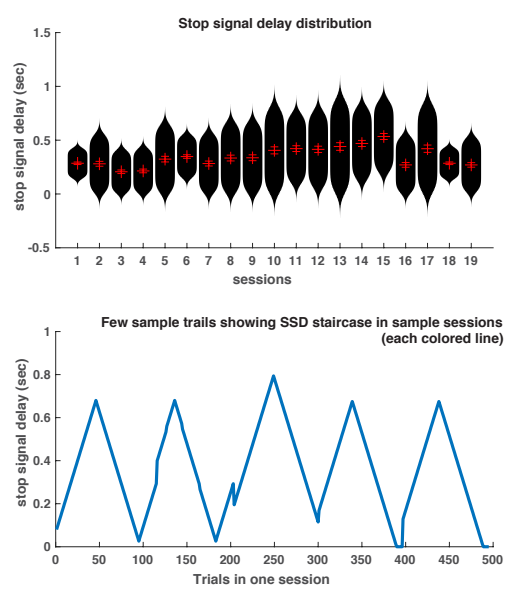

We collected our data on day basis and it was for 19 days in subject $\mathrm{J}$ and 13 days in

The SSD starting point is a random floating value between 0 and 0.1 secs. Below in the top subplot of the figures, we show SSD distribution plots for each session in both subjects. In the bottom subplot, we show that a representative session's SSD (current value) trail oscillate around a mean SSD value (SSD-50, as evident from the triangular curves) through trials, both in subject $\mathrm{J}$ and $\mathrm{T}$, suggesting they stabilize and converge. The starting point of the trail is when the neural recording started for the day. (Note that the start of neural recording isn't exactly the start of the task session for the day).

In the next figure, the top panel (A) shows the violin distributions of SSRT for subjects J 702 and $\mathrm{T}$ across recorded sessions, whereas the bottom (B) panel draws it out explicitly as a 703 function of session. 
bioRxiv preprint doi: https://doi.org/10.1101/2020.04.23.057455; this version posted April 25, 2020. The copyright holder for this preprint (which

was not certified by peer review) is the author/funder, who has granted bioRxiv a license to display the preprint in perpetuity. It is made available under aCC-BY-NC-ND 4.0 International license.

704

705

706

707

708

709

710

711

712

713

714

715

716

717
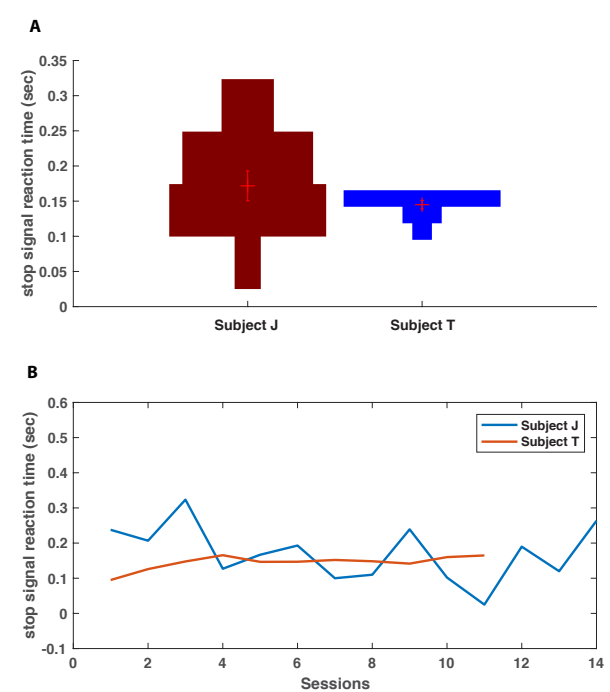

\section{DATA ACCESSIBILITY:}

All data will be available on the data section of the Hayden Lab website (www.ha ydenlab.com/datacode.html, https://doi.org/10.6084/m9.figshare.7865297.v1) 


\section{REFERENCES}

719 Aron AR. 2007. The neural basis of inhibition in cognitive control. The neuroscientist.

720 13(3):214-228.

721 Aron AR, Poldrack RA. 2006. Cortical and subcortical contributions to stop signal

722 response inhibition: role of the subthalamic nucleus. Journal of Neuroscience.

723 26(9):2424-2433.

724 Averbeck BB, Latham PE, Pouget A. 2006a. Neural correlations, population coding and

725 computation. Nature Reviews Neuroscience. 7(5):358.

726 Averbeck BB, Latham PE, Pouget A. 2006b. Neural correlations, population coding and

727 computation. Nature Reviews Neuroscience. 7(5):358.

728 Azab, H. and Hayden, B.Y., 2017. Correlates of decisional dynamics in the dorsal

729 anterior cingulate cortex. PLoS biology, 15(11), p.e2003091.

730 Azab, H. and Hayden, B.Y., 2018. Correlates of economic decisions in the dorsal and

731 subgenual anterior cingulate cortices. European Journal of Neuroscience, 47(8),

732 pp.979-993.

733 Balasubramani PP, Moreno-Bote R, Hayden B. 2018. Using a simple neural network to 734 delineate some principles of distributed economic choice. Frontiers in Computational 735 Neuroscience. 12:22.

736 Balasubramani PP, Pesce MC, Hayden BY. 2019 Dec 5. Activity in orbitofrontal 737 neuronal ensembles reflects inhibitory control. Eur J Neurosci. doi:10.1111/ejn.14638.

738 Baumeister RF, Heatherton TF. 1996. Self-regulation failure: An overview. Psychological 739 inquiry. $7(1): 1-15$.

740 Baumeister RF, Newman LS. 1994. Self-Regulation of Cognitive Inference and Decision

741 Processes. Pers Soc Psychol Bull. 20(1):3-19. doi:10.1177/0146167294201001.

742 Berkman E, Hutcherson C, Livingston JL, Kahn LE, Inzlicht M. 2016. Self-control as

743 value-based choice.

744 Blanchard, T.C., Wilke, A. and Hayden, B.Y., 2014. Hot-hand bias in rhesus monkeys.

745 Journal of Experimental Psychology: Animal Learning and Cognition, 40(3), p.280.

746 Blanchard TC, Hayden BY. 2015. Monkeys are more patient in a foraging task than in a 747 standard intertemporal choice task. PloS one. 10(2):e0117057.

748 Blanchard TC, Strait CE, Hayden BY. 2015. Ramping ensemble activity in dorsal 749 anterior cingulate neurons during persistent commitment to a decision. Journal of 750 neurophysiology. 114(4):2439-2449.

751 Brainard DH, Vision S. 1997. The psychophysics toolbox. Spatial vision. 10:433-436. 
752 Braver TS. 2012. The variable nature of cognitive control: a dual mechanisms

753 framework. Trends in cognitive sciences. 16(2):106-113.

754 Braver TS, Gray JR, Burgess GC. 2007. Explaining the many varieties of working 755 memory variation: Dual mechanisms of cognitive control. Variation in working

756 memory.:76-106.

757 Bryden DW, Roesch MR. 2015. Executive control signals in orbitofrontal cortex during 758 response inhibition. The Journal of neuroscience. 35(9):3903-3914.

759 Calhoun AJ, Hayden BY. 2015. The foraging brain. Current Opinion in Behavioral

760 Sciences. 5:24-31. doi:10.1016/j.cobeha.2015.07.003.

761 Carver CS. 2005. Impulse and constraint: Perspectives from personality psychology, 762 convergence with theory in other areas, and potential for integration. Personality and 763 social psychology review. 9(4):312-333.

764 Chen X, Scangos KW, Stuphorn V. 2010. Supplementary motor area exerts proactive 765 and reactive control of arm movements. Journal of Neuroscience. 30(44):14657-14675.

766 Chikazoe J, Jimura K, Hirose S, Yamashita K, Miyashita Y, Konishi S. 2009. Preparation 767 to inhibit a response complements response inhibition during performance of a stop768 signal task. Journal of Neuroscience. 29(50):15870-15877.

769 Chudasama Y, Kralik JD, Murray EA. 2006. Rhesus monkeys with orbital prefrontal 770 cortex lesions can learn to inhibit prepotent responses in the reversed reward 771 contingency task. Cerebral cortex. 17(5):1154-1159.

772 Cisek P. 2012. Making decisions through a distributed consensus. Current opinion in 773 neurobiology. 22(6):927-936.

774 Cisek P, Kalaska JF. 2010. Neural mechanisms for interacting with a world full of action

775 choices. Annual review of neuroscience. 33:269-298.

776 Cisek P, Pastor-Bernier A. 2014. On the challenges and mechanisms of embodied 777 decisions. Phil Trans R Soc B. 369(1655):20130479.

778 Cornelissen FW, Peters EM, Palmer J. 2002. The Eyelink Toolbox: eye tracking with

779 MATLAB and the Psychophysics Toolbox. Behavior Research Methods. 34(4):613-617.

780 Cunningham JP, Byron MY. 2014. Dimensionality reduction for large-scale neural 781 recordings. Nature neuroscience. 17(11):1500.

782 David, S. V., \& Hayden, B. Y. (2012). Neurotree: A collaborative, graphical database of 783 the academic genealogy of neuroscience. PloS one, 7(10).

784 Dias R, Robbins TW, Roberts AC. 1996. Dissociation in prefrontal cortex of affective 785 and attentional shifts. Nature. 380(6569):69-72. 
786 Eagle DM, Baunez C, Hutcheson DM, Lehmann O, Shah AP, Robbins TW. 2007. Stopsignal reaction-time task performance: role of prefrontal cortex and subthalamic

788 nucleus. Cerebral cortex. 18(1):178-188.

789 Eisenreich BR, Akaishi R, Hayden BY. 2017. Control without Controllers: Toward a

790 Distributed Neuroscience of Executive Control. Journal of cognitive neuroscience.

791 Farashahi, S., Azab, H., Hayden, B. and Soltani, A., 2018. On the flexibility of basic risk

792 attitudes in monkeys. Journal of Neuroscience, 38(18), pp.4383-4398.

793 Feierstein CE, Quirk MC, Uchida N, Sosulski DL, Mainen ZF. 2006. Representation of

794 spatial goals in rat orbitofrontal cortex. Neuron. 51(4):495-507.

795 FUJITA K. 2011. On Conceptualizing Self-Control as More Than the Effortful Inhibition

796 of Impulses. Pers soc psychol rev (Print). 15(4):352-366.

797 Ghods-Sharifi S, Haluk DM, Floresco SB. 2008. Differential effects of inactivation of the

798 orbitofrontal cortex on strategy set-shifting and reversal learning. Neurobiology of

799 learning and memory. 89(4):567-573.

800 Gochin PM, Colombo M, Dorfman GA, Gerstein GL, Gross CG. 1994. Neural ensemble 801 coding in inferior temporal cortex. Journal of neurophysiology. 71(6):2325-2337.

802 Hampshire A, Sharp DJ. 2015. Contrasting network and modular perspectives on

803 inhibitory control. Trends in cognitive sciences. 19(8):445-452.

804 Hanes DP, Patterson WF, Schall JD. 1998. Role of frontal eye fields in countermanding 805 saccades: visual, movement, and fixation activity. Journal of neurophysiology.

806 79(2):817-834.

807 Hanes DP, Schall JD. 1995. Countermanding saccades in macaque. Visual

808 neuroscience. 12(5):929-937.

809 Hayden, B. Y., Pearson, J. M., \& Platt, M. L. 2011. Neuronal basis of sequential foraging 810 decisions in a patchy environment. Nature neuroscience, 14(7), 933.

811 Hayden B, Gallant J. 2013. Working memory and decision processes in visual area v4.

812 Frontiers in neuroscience. 7:18.

813 Hayden BY. 2016. Time discounting and time preference in animals: a critical review.

814 Psychonomic Bulletin \& Review. 23(1):39-53.

815 Hayden BY. 2018. Economic choice: the foraging perspective. Current Opinion in 816 Behavioral Sciences. 24:1-6.

817 Hayden BY, Moreno-Bote R. 2018. A neuronal theory of sequential economic choice. 818 Brain and Neuroscience Advances. 2:2398212818766675.

819 doi:10.1177/2398212818766675.

820 Haykin S, Network N. 2004. A comprehensive foundation. Neural Networks. 2(2004):41. 
821 Heilbronner, S.R. and Hayden, B.Y., 2016. The description-experience gap in risky

822 choice in nonhuman primates. Psychonomic bulletin \& review, 23(2), pp.593-600.

823 Hillman KL, Bilkey DK. 2010. Neurons in the rat anterior cingulate cortex dynamically

824 encode cost-benefit in a spatial decision-making task. Journal of Neuroscience.

825 30(22):7705-7713.

826 Horn NR, Dolan M, Elliott R, Deakin JFW, Woodruff PWR. 2003. Response inhibition

827 and impulsivity: an fMRI study. Neuropsychologia. 41(14):1959-1966.

828 Hunt LT, Hayden BY. 2017. A distributed, hierarchical and recurrent framework for

829 reward-based choice. Nat Rev Neurosci. 18(3):172-182. doi:10.1038/nrn.2017.7.

830 lacono WG, Malone SM, McGue M. 2008. Behavioral disinhibition and the development

831 of early-onset addiction: common and specific influences. Annu Rev Clin Psychol.

832 4:325-348.

833 Inzlicht M, Schmeichel BJ, Macrae CN. 2014. Why self-control seems (but may not be)

834 limited. Trends in cognitive sciences. 18(3):127-133.

835 Ito S, Stuphorn V, Brown JW, Schall JD. 2003. Performance monitoring by the anterior

836 cingulate cortex during saccade countermanding. Science. 302(5642):120-122.

837 Iversen SD, Mishkin M. 1970. Perseverative interference in monkeys following selective 838 lesions of the inferior prefrontal convexity. Experimental Brain Research. 11(4):376-386.

839 James W. 1890. The principles of psychology, Vol. 2. Henry Holt and Company.

840 Kable JW, Glimcher PW. 2007. The neural correlates of subjective value during

841 intertemporal choice. Nature neuroscience. 10(12):1625.

842 Kacelnik A, Vasconcelos M, Monteiro T, Aw J. 2011. Darwin's "tug-of-war" vs.

843 starlings' "horse-racing": how adaptations for sequential encounters drive simultaneous

844 choice. Behavioral Ecology and Sociobiology. 65(3):547-558.

845 Krajbich I, Armel C, Rangel A. 2010. Visual fixations and the computation and

846 comparison of value in simple choice. Nature neuroscience. 13(10):1292.

847 Lim S-L, O'Doherty JP, Rangel A. 2011. The decision value computations in the vmPFC 848 and striatum use a relative value code that is guided by visual attention. Journal of

849 Neuroscience. 31(37):13214-13223.

850 Logan GD. 1994. On the ability to inhibit thought and action: A users' guide to the stop 851 signal paradigm.

852 Logan GD, Cowan WB. 1984. On the ability to inhibit thought and action: A theory of an 853 act of control. Psychological review. 91(3):295. 
854 Logan GD, Yamaguchi M, Schall JD, Palmeri TJ. 2015. Inhibitory control in mind and 855 brain 2.0: blocked-input models of saccadic countermanding. Psychological review. 856 122(2):115.

857 Majid DA, Cai W, Corey-Bloom J, Aron AR. 2013. Proactive selective response 858 suppression is implemented via the basal ganglia. Journal of Neuroscience.

859 33(33):13259-13269.

860 Mante V, Sussillo D, Shenoy KV, Newsome WT. 2013. Context-dependent computation 861 by recurrent dynamics in prefrontal cortex. Nature. 503(7474):78.

862 McClure SM, Laibson DI, Loewenstein G, Cohen JD. 2004. Separate neural systems 863 value immediate and delayed monetary rewards. Science. 306(5695):503-507.

864 McGuire JT, Kable JW. 2015. Medial prefrontal cortical activity reflects dynamic re865 evaluation during voluntary persistence. Nature neuroscience. 18(5):760-766.

866 Meyers EM, Freedman DJ, Kreiman G, Miller EK, Poggio T. 2008. Dynamic population 867 coding of category information in inferior temporal and prefrontal cortex. Journal of 868 neurophysiology. 100(3):1407-1419.

869 Mischel W, Cantor N, Feldman S. 1996. Principles of self-regulation: The nature of 870 willpower and self-control. In: Social psychology: Handbook of basic principles. New 871 York, NY, US: Guilford Press. p. 329-360.

872 Mishkin M. 1964. Perseveration of central sets after frontal lesions in monkeys. The 873 frontal granular cortex and behavior.:219-241.

874 Mobbs D, Trimmer PC, Blumstein DT, Dayan P. 2018. Foraging for foundations in 875 decision neuroscience: insights from ethology. Nat Rev Neurosci. 19(7):419-427. 876 doi:10.1038/s41583-018-0010-7.

877 Morcos AS, Barrett DG, Rabinowitz NC, Botvinick M. 2018. On the importance of single 878 directions for generalization. arXiv preprint arXiv:180306959.

879 MURAVEN M, BAUMEISTER RF. 2000. Self-regulation and depletion of limited

880 resources: Does self-control resemble a muscle? Psychol bull. 126(2):247-259.

881 Nestler EJ, Barrot M, DiLeone RJ, Eisch AJ, Gold SJ, Monteggia LM. 2002.

882 Neurobiology of depression. Neuron. 34(1):13-25.

883 Ojeda A, Murphy RA, Kacelnik A. 2018. Paradoxical choice in rats: subjective valuation 884 and mechanism of choice. Behavioural processes.

885 Padoa-Schioppa C. 2011. Neurobiology of economic choice: a good-based model.

886 Annual review of neuroscience. 34:333.

887 Padoa-Schioppa C. 2013. Neuronal origins of choice variability in economic decisions. 888 Neuron. 80(5):1322-1336. 
889 Padoa-Schioppa C, Assad JA. 2006. Neurons in the orbitofrontal cortex encode 890 economic value. Nature. 441(7090):223-226.

891 Paxinos G, Watson C. 2006. The Rat Brain in Stereotaxic Coordinates: Hard Cover

892 Edition. Elsevier.

893 Pearson JM, Watson KK, Platt ML. 2014. Decision making: the neuroethological turn.

894 Neuron. 82(5):950-965.

895 Pirrone, A., Azab, H., Hayden, B. Y., Stafford, T., \& Marshall, J. A. (2018). Evidence for

896 the speed-value trade-off: Human and monkey decision making is magnitude sensitive.

897 Decision, 5(2), 129.

898 Raghuraman AP, Padoa-Schioppa C. 2014. Integration of multiple determinants in the 899 neuronal computation of economic values. Journal of Neuroscience. 34(35):1158390011603.

901 Rich EL, Stoll FM, Rudebeck PH. 2017. Linking dynamic patterns of neural activity in 902 orbitofrontal cortex with decision making. Current opinion in neurobiology. 49:24-32.

903 Rich EL, Wallis JD. 2016. Decoding subjective decisions from orbitofrontal cortex.

904 Nature neuroscience. 19(7):973.

905 Rigotti M, Barak O, Warden MR, Wang X-J, Daw ND, Miller EK, Fusi S. 2013. The 906 importance of mixed selectivity in complex cognitive tasks. Nature. 497(7451):585-590.

907 Roberts AC, Wallis JD. 2000. Inhibitory control and affective processing in the prefrontal 908 cortex: neuropsychological studies in the common marmoset. Cerebral cortex.

909 10(3):252-262.

910 Roesch MR, Taylor AR, Schoenbaum G. 2006. Encoding of time-discounted rewards in 911 orbitofrontal cortex is independent of value representation. Neuron. 51(4):509-520.

912 Rudebeck PH, Murray EA. 2014. The orbitofrontal oracle: cortical mechanisms for the

913 prediction and evaluation of specific behavioral outcomes. Neuron. 84(6):1143-1156.

914 Rumelhart DE, McClelland JL, PDP Research Group. 1988. Parallel distributed 915 processing. IEEE.

916 Rumelhart DE, McClelland JL, Williams RJ. 1986. Parallel recognition in modern

917 computers. processing: Explorations in the microstructure of cognition. 1.

918 Sakagami M, Pan X. 2007. Functional role of the ventrolateral prefrontal cortex in 919 decision making. Current opinion in neurobiology. 17(2):228-233.

920 Schall JD. 1991. Neuronal activity related to visually guided saccades in the frontal eye 921 fields of rhesus monkeys: comparison with supplementary eye fields. Journal of 922 neurophysiology. 66(2):559-579. 
923 Schall JD, Stuphorn V, Brown JW. 2002. Monitoring and control of action by the frontal

924 lobes. Neuron. 36(2):309-322.

925 Schoenbaum G, Roesch MR, Stalnaker TA, Takahashi YK. 2009. A new perspective on 926 the role of the orbitofrontal cortex in adaptive behaviour. Nature Reviews Neuroscience.

927 10(12):885-892.

928 Schoenbaum G, Setlow B, Nugent SL, Saddoris MP, Gallagher M. 2003. Lesions of 929 orbitofrontal cortex and basolateral amygdala complex disrupt acquisition of odor930 guided discriminations and reversals. Learning \& Memory. 10(2):129-140.

931 Shapiro MS, Siller S, Kacelnik A. 2008. Simultaneous and sequential choice as a 932 function of reward delay and magnitude: normative, descriptive and process-based 933 models tested in the European starling (Sturnus vulgaris). Journal of Experimental 934 Psychology: Animal Behavior Processes. 34(1):75.

935 Shenhav A. 2017. The perils of losing control: Why self-control is not just another value936 based decision. Psychological Inquiry.

937 Shenhav A, Botvinick MM, Cohen JD. 2013. The expected value of control: an 938 integrative theory of anterior cingulate cortex function. Neuron. 79(2):217-240.

939 Sleezer, B. J., \& Hayden, B. Y. (2016). Differential contributions of ventral and dorsal 940 striatum to early and late phases of cognitive set reconfiguration. Journal of cognitive 941 neuroscience, 28(12), 1849-1864.

942

943

944

945

946

947

Sleezer, B.J., Castagno, M.D. and Hayden, B.Y., 2016. Rule encoding in orbitofrontal cortex and striatum guides selection. Journal of Neuroscience, 36(44), pp.11223-11237.

Sleezer, B.J., LoConte, G.A., Castagno, M.D. and Hayden, B.Y., 2017. Neuronal responses support a role for orbitofrontal cortex in cognitive set reconfiguration. European Journal of Neuroscience, 45(7), pp.940-951.

949 Stalnaker TA, Cooch NK, Schoenbaum G. 2015. What the orbitofrontal cortex does not 950 do. Nature neuroscience. 18(5):620-627.

951 Stephens DW, Anderson D. 2001. The adaptive value of preference for immediacy: 952 when shortsighted rules have farsighted consequences. Behavioral Ecology. 12(3):330953339.

954 Stephens DW, Krebs JR. 1986. Foraging theory. Princeton University Press.

955 Stokes MG. 2015. 'Activity-silent'working memory in prefrontal cortex: a dynamic 956 coding framework. Trends in cognitive sciences. 19(7):394-405.

957 Stokes MG, Kusunoki M, Sigala N, Nili H, Gaffan D, Duncan J. 2013. Dynamic coding 958 for cognitive control in prefrontal cortex. Neuron. 78(2):364-375.

959 Strait CE, Blanchard TC, Hayden BY. 2014. Reward value comparison via mutual 960 inhibition in ventromedial prefrontal cortex. Neuron. 82(6):1357-1366. 
961 Strait CE, Sleezer BJ, Blanchard TC, Azab H, Castagno MD, Hayden BY. 2016.

962 Neuronal selectivity for spatial positions of offers and choices in five reward regions.

963 Journal of neurophysiology. 115(3):1098-1111.

964 Strait CE, Sleezer BJ, Hayden BY. 2015. Signatures of value comparison in ventral

965 striatum neurons. PLoS Biol. 13(6):e1002173.

966 Stuphorn V, Brown JW, Schall JD. 2010. Role of supplementary eye field in saccade

967 initiation: executive, not direct, control. Journal of neurophysiology. 103(2):801-816.

968 Stuphorn V, Emeric EE. 2012. Proactive and reactive control by the medial frontal

969 cortex. Frontiers in Neuroengineering. 5.

970 Stuphorn V, Taylor TL, Schall JD. 2000. Performance monitoring by the supplementary

971 eye field. Nature. 408(6814):857-860.

972 Thomas E, Van Hulle MM, Vogel R. 2001. Encoding of categories by noncategory-

973 specific neurons in the inferior temporal cortex. Journal of cognitive neuroscience.

974 13(2):190-200.

975 Vasconcelos M, Monteiro T, Aw J, Kacelnik A. 2010. Choice in multi-alternative

976 environments: a trial-by-trial implementation of the sequential choice model.

977 Behavioural processes. 84(1):435-439.

978 Verbruggen F, Logan GD. 2008. Response inhibition in the stop-signal paradigm.

979 Trends in cognitive sciences. 12(11):418-424.

980 Volkow ND, Wang G-J, Fowler JS, Tomasi D, Telang F. 2011. Addiction: beyond 981 dopamine reward circuitry. Proceedings of the National Academy of Sciences.

982 108(37):15037-15042.

983 Wallis JD. 2007. Orbitofrontal cortex and its contribution to decision-making. Annu Rev 984 Neurosci. 30:31-56.

985 Wang MZ, Hayden BY. 2017. Reactivation of associative structure specific outcome

986 responses during prospective evaluation in reward-based choices. Nature

987 Communications. 8:ncomms15821.

988 Werbos P. 1974. Beyond regression: New tools for prediction and analysis in the 989 behavioral sciences.

990 Wilson RC, Takahashi YK, Schoenbaum G, Niv Y. 2014. Orbitofrontal cortex as a 991 cognitive map of task space. Neuron. 81(2):267-279.

992 Xie Y, Nie C, Yang T. 2018. Covert shift of attention modulates the value encoding in 993 the orbitofrontal cortex. eLife. 7:e31507.

994 Yoo, S.B.M. and Hayden, B.Y., 2018. Economic choice as an untangling of options into 995 actions. Neuron, 99(3), pp.434-447. 
996 Yoo, S.B.M. and Hayden, B.Y., 2020. The Transition from Evaluation to Selection

997 Involves Neural Subspace Reorganization in Core Reward Regions. Neuron, 105(4),

998 pp.712-724.

999 Yoo, S.B.M., Sleezer, B.J. and Hayden, B.Y., 2018. Robust encoding of spatial

1000 information in orbitofrontal cortex and striatum. Journal of cognitive neuroscience,

1001 30(6), pp.898-913.

1002 Zemel RS, Dayan P, Pouget A. 1998. Probabilistic interpretation of population codes.

1003 Neural computation. 10(2):403-430.

1004 Zhang Z, Cheng Z, Lin Z, Nie C, Yang T. 2018. A neural network model for the

1005 orbitofrontal cortex and task space acquisition during reinforcement learning. PLOS

1006 Computational Biology. 14(1):e1005925.

1007 